\title{
Behandlung von Patienten aus EU- und EFTA-Staaten: europäische Versicherungskarte und Ansprüche
}

Lucia Rabia, Rechtsdienst FMH

Am 1. Juni 2004 sind einige technische Anpassungen im Rahmen des Freizügigkeitsabkommens und des EFTA-Übereinkommens im Bereich der Krankenversicherung in Kraft getreten. Im Zentrum steht die Einführung einer europäischen Versicherungskarte. Die Neuerungen gelten aber nur für die von den bilateralen Abkommen erfassten Länder, also nicht für die 10 neuen Mitgliedstaaten im Rahmen der EUOst-Erweiterung.

Unverändert bleiben der anwendbare Tarif und die administrative Abwicklung. Verwiesen sei auf die praktischen Informationen von R. Gmür [1].

\section{Einführung der europäischen Kranken- versicherungskarte}

Auf den 1. Juni 2004 wurde in den meisten EU- und EFTA-Staaten die europäische Krankenversicherungskarte eingeführt. Sie ersetzt das bisherige Formular E 111. Die europäische Krankenversicherungskarte hat das Format einer

Abbildung 1

Europäische Versicherungskarte.

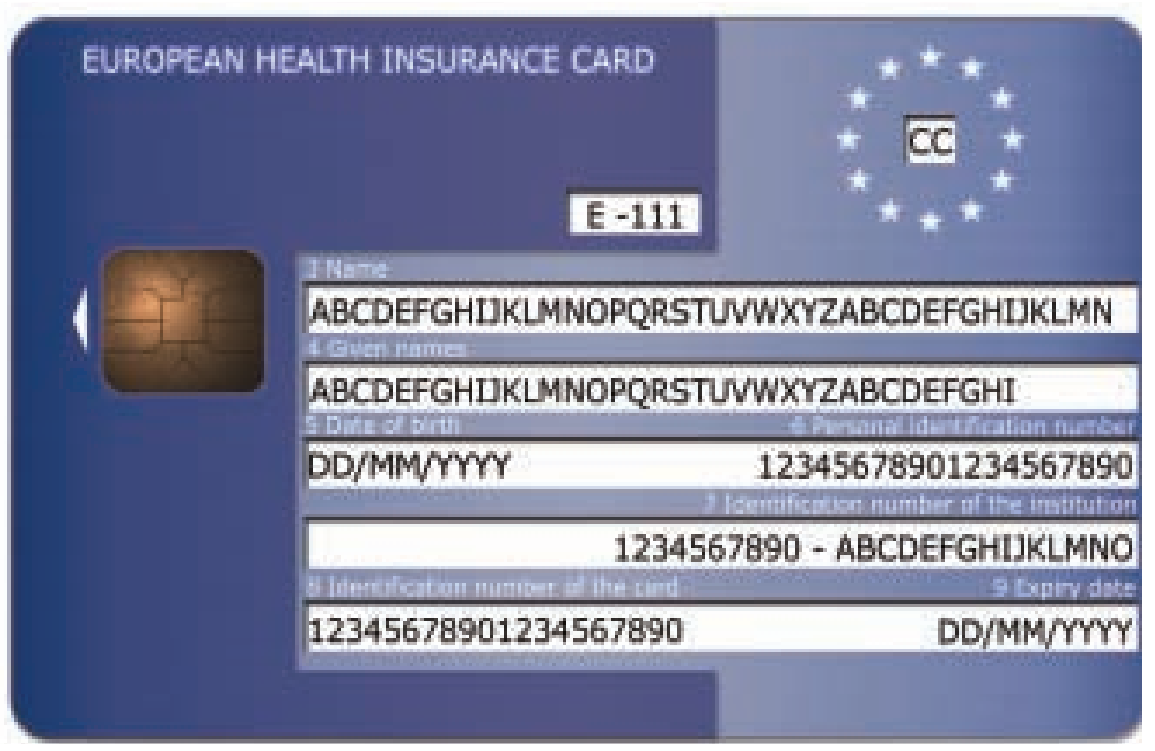

Kreditkarte (Abb. 1). Sie ist keine Gesundheitskarte, denn sie enthält keine medizinischen Daten.

Eine provisorische Ersatzbescheinigung mit befristeter Gültigkeit wird im Falle von ausserordentlichen Umständen ausgestellt (z.B. Verlust der Karte oder kurzfristige Abreise).

Staaten, denen für die Einführung der Karte eine Übergangsfrist bis längstens Ende 2005 gewährt wurde, stellen statt dessen ein neues Formular E 111 aus, welches die gleichen Angaben enthält wie die europäische Krankenversicherungskarte.

\section{Auswirkungen für Sie in der Praxis}

Seit dem 1. Juni 2004 haben die Versicherten aus den EU-/EFTA-Staaten für Behandlungen in der Schweiz den Leistungserbringern als Bescheinigung entweder die europäische Krankenversicherungskarte, die provisorische Ersatzbescheinigung oder das neue Formular E 111 vorzulegen.

\section{Angleichung der Ansprüche}

Für den Umfang des Leistungsanspruchs bei vorübergehendem Auslandsaufenthalt spielt der Status der Patientin (Touristin, Rentner, Arbeitsloser, Studentin usw.) künftig keine Rolle mehr. Alle versicherten Personen haben bei Krankheit und Mutterschaft während eines Aufenthalts im Gebiet eines anderen EU-/EFTA-Staates Anspruch auf diejenige Behandlung, die sich unter Berücksichtigung der voraussichtlichen Aufenthaltsdauer als medizinisch notwendig erweist. Es wird nicht mehr zwischen unverzüglich erforderlichen Behandlungen und erforderlichen Behandlungen unterschieden.

Weiterhin nicht abgedeckt ist der Fall, in dem der Patient in die Schweiz kommt, um sich medizinisch behandeln $\mathrm{zu}$ lassen. In diesem Fall werden die Kosten von seiner Krankenversicherung nur übernommen, wenn diese ihre Zustimmung erteilt hat, also das Formular E 112 ausgestellt hat. 


\section{Auswirkungen für Sie in der Praxis}

Sie müssen bei der Behandlung von Patienten aus den EU-/EFTA-Staaten nicht mehr darauf achten, welchen Status diese haben. Insbesondere bei Touristen müssen Sie sich nicht mehr auf eine notfallmässige Behandlung beschränken. Sie können die Behandlung vielmehr darauf ausrichten, dass die Patientin ihren ursprünglich geplanten Aufenthalt fortsetzen kann und nicht eigens nach Hause zurückkehren muss, um sich behandeln zu lassen.

\section{Vereinfachung der E-Formulare}

Mit den in Kraft getretenen Änderungen wurden einige für die Krankenversicherung geltenden
E-Formulare angepasst oder aufgehoben. Wer sich für die Details interessiert, findet diese im BAG-Kreisschreiben.

Das vollständige BAG-Kreisschreiben EU 04/3 können Sie unter folgender Adresse abrufen: www.sozialversicherungen.admin.ch $\rightarrow$ $\mathrm{KV} \rightarrow \mathrm{EU} \rightarrow$ Kantone.

Die E-Formulare inkl. Muster der europäischen Versicherungskarte finden Sie unter www.sozialversicherungen.admin.ch $\rightarrow$ International $\rightarrow$ Formulare $\rightarrow \mathrm{CH} / \mathrm{EU} \rightarrow \mathrm{E}$ 104-128.

Literatur

Gmür R. Behandlung von Patienten aus EU- und EFTA-Staaten: Abrechnung und Tarife. Schweiz Ärztezeitung 2003;84(1/2):15-6.

\section{Traitement de patients en provenance des Etats de l'UE et de l'AELE: carte européenne d'assurance-maladie et droits des patients}

Le $1^{\text {er }}$ juin 2004, quelques adaptations techniques sont entrées en vigueur dans le cadre de l'accord sur la libre circulation des personnes et de la convention AELE, adaptations concernant le domaine de l'assurance-maladie. L'introduction d'une carte européenne d'assurance-maladie en est l'objet principal. Les innovations ne valent toutefois que pour les pays concernés par les accords bilatéraux; elles ne s'appliquent donc pas aux ressortissants des 10 nouveaux Etats devenus membres dans le cadre de l'élargissement de l'UE vers l'Est.

Le tarif applicable et la procédure administrative demeurent inchangés; vous trouverez des informations pratiques dans un article de R. Gmür consacré à ce sujet [1].

\section{Introduction de la carte européenne d'assurance-maladie}

La carte européenne d'assurance-maladie a été introduite dans la plupart des Etats de l'UE et de
l'AELE avec effet au $1^{\text {er }}$ juin 2004. Elle remplace le formulaire $\mathrm{E} 111$ utilisé jusqu'à présent. Cette carte a le format d'une carte de crédit (fig. 1). Il ne s'agit pas d'une carte-santé car elle ne contient pas de données médicales.

Un certificat provisoire de remplacement de validité limitée sera établi dans des circonstances exceptionnelles (p.ex. perte de la carte ou départ dans un délai trop court).

Les Etats bénéficiant d'une période transitoire jusqu'à fin 2005 au plus tard pour l'introduction de la carte continueront à délivrer un nouveau formulaire E 111 contenant les mêmes indications que la carte européenne d'assurancemaladie.

\section{Conséquences pour votre cabinet médical} Depuis le $1^{\text {er }}$ juin 2004, les assurés des Etats de l'UE/de l'AELE doivent, lors de traitements en Suisse, présenter aux fournisseurs de prestations la carte européenne d'assurance-maladie, le certificat provisoire de remplacement ou le nouveau formulaire E 111. 
Figure 1

Carte européenne d'assurance-maladie.

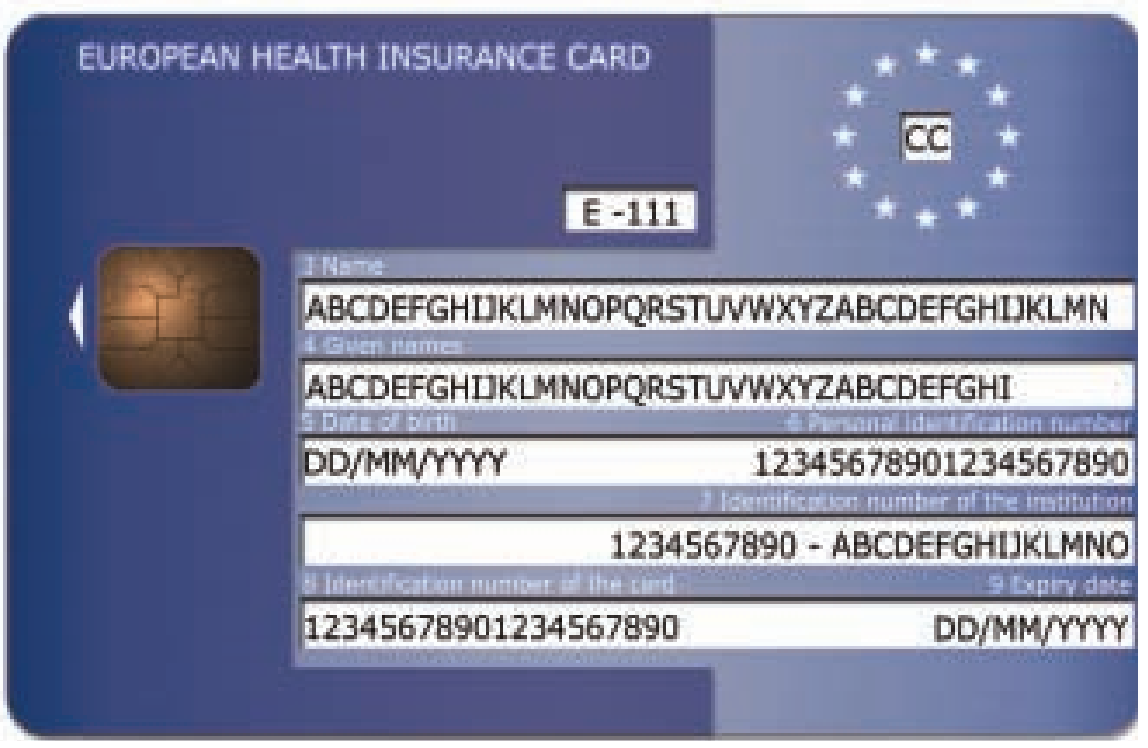

\section{Alignement des droits}

Désormais, le statut du patient (touriste, rentier, chômeur, étudiant, etc.) ne joue plus aucun rôle pour l'étendue des prestations lors d'un séjour momentané à l'étranger. En cas de maladie et de maternité toutes les personnes assurées ont droit, pendant un séjour sur le territoire d'un autre Etat de l'UE/de l'AELE au traitement qui s'avère médicalement nécessaire, compte tenu de la durée prévue du séjour. La distinction entre traitements immédiatement nécessaires et traitements nécessaires n'existe plus.

En revanche, le patient qui vient en Suisse dans l'intention d'y suivre un traitement médical n'est toujours pas couvert, et les coûts ne sont pris en charge par son assurance-maladie qu'avec le consentement préalable de celle-ci, donc uniquement si elle a délivré auparavant le formulaire E 112.

\section{Conséquences pour votre cabinet médical}

Vous ne devez plus tenir compte du statut des patients en provenance des Etats de l'UE/ de l'AELE. Concernant en particulier les touristes, vous ne devez plus vous limiter à un traitement d'urgence. Vous pourrez, au contraire, prévoir un traitement permettant au patient de poursuivre le séjour prévu à l'origine et ne l'obligeant plus à rentrer chez lui pour se faire soigner.

\section{Simplification des formulaires $E$}

Certains points des formulaires $\mathrm{E}$, valables pour l'assurance-maladie, ont été adaptés ou supprimés dans la foulée des modifications entrées en vigueur. Vous trouverez des informations détaillées à ce sujet dans la lettre-circulaire UE 04/3 de l'OFSP.

Voici le lien pour obtenir cette lettre-circulaire complète à l'écran: www.sozialversicherungen.admin.ch $\rightarrow$ AMAL $\rightarrow$ UE $\rightarrow$ Cantons.

Vous trouvez les formulaires E ainsi qu'un modèle de carte européenne d'assurance-maladie sous www.sozialversicherungen.admin.ch $\rightarrow$ International $\rightarrow$ Formulaires $\rightarrow \mathrm{CH} / \mathrm{UE} \rightarrow$ E 104-128.

\section{Référence}

1 Gmür R. Traitements prodigués à des ressortissants des Etats de l'UE/de l'AELE: facturation et tarifs. Bull Méd Suisses 2003;84(1/2):16-7. 